\title{
GAC - the Criterion for Global Avalanche Characteristics of Cryptographic Functions
}

\author{
Xian-Mo Zhang \\ (The University of Wollongong, Wollongong, NSW 2522, Australia \\ xianmo@cs.uow.edu.au) \\ Yuliang Zheng \\ (Monash University, Melbourne, VIC 3199, Australia \\ yzheng@fcit.monash.edu.au)
}

\begin{abstract}
We show that some widely accepted criteria for cryptographic functions, including the strict avalanche criterion (SAC) and the propagation criterion, have various limitations in capturing properties of vital importance to cryptographic algorithms, and propose a new criterion called GAC to measure the global avalanche characteristics of cryptographic functions. We also introduce two indicators related to the new criterion, one forecasts the sum-of-squares while the other the absolute avalanche characteristics of a function. Lower and upper bounds on the two indicators are derived, and two methods are presented to construct cryptographic functions that achieve nearly optimal global avalanche characteristics.
\end{abstract}

Category: E. 3

\section{Why the GAC}

In 1985, Webster and Tavares introduced the concept of the strict avalanche criterion ( $S A C$ ) when searching for principles for designing DES-like data encryption algorithms [Web85, WT86]. A function is said to satisfy the SAC if complementing a single bit results in the output of the function being complemented with a probability of a half. More formally, let $V_{n}$ denote the vector space of $n$ tuples of elements from $\mathrm{GF}(2)$, a function $f$ on $V_{n}$, a mapping from $V_{n}$ into $\mathrm{GF}(2)$, is said to satisfy the SAC if for any $n$-bit vector $\alpha$ with $W(\alpha)=1$, where $W(\cdot)$ denotes the Hamming weight, $f(x) \oplus f(x \oplus \alpha)$ assumes the values zero and one an equal number of times, namely $f(x) \oplus f(x \oplus \alpha)$ is a balanced function on $V_{n}$, where $\oplus$ denotes the addition in $\mathrm{GF}(2)$.

The SAC was generalized in one direction by Forré in [For89]. Forré defines that a function $f$ satisfies the SAC of order $k$ if a partial function obtained by keeping any $k$ input bits to $f$ constant still satisfies the SAC. Enumerating functions satisfying the higher order SAC is an interesting combinatorial problem and various results on this topic have been obtained over the past years (see for instance [Llo90, Llo92, Mit90]). In another direction, the SAC has been generalized by Adams and Tavares [AT90] and independently by Preneel et al [PLL $\left.{ }^{+} 91\right]$ to what is now called the propagation criterion. A function $f$ on $V_{n}$ is said to satisfy the propagation criterion with respect to a vector $\alpha \in V_{n}$ if $f(x) \oplus f(x \oplus \alpha)$ is balanced, and to satisfy the propagation criterion of degree $k$ if it satisfies the propagation criterion with respect to all nonzero vectors whose Hamming weight is at most $k$. In informal terms, $f$ satisfies the propagation criterion of degree $k$ if complementing $k$ or less bits results in the output of $f$ being complemented with a probability of a half. We note that functions satisfying the propagation criterion 
of degree $n$ coincide with bent functions, an important combinatorial structure discovered by Rothaus [Rot76]. A combination of the two generalizations has also been studied in [PLL +91, PGV91].

The SAC and its various generalizations are very important concepts in designing cryptographic functions employed by data encryption algorithms and one-way hashing functions. As is shown below, however, these concepts all have their limitations in capturing some of the vital characteristics required by a cryptographically strong function. The following concept of linear structure will be useful in our discussions. Given a function $f$ on $V_{n}$ and a vector $\alpha \in V_{n}$, the vector is said to be a linear structure of $f$ if $f(x) \oplus f(x \oplus \alpha)$ is a constant. An affine function $f(x)=a_{1} x_{1} \oplus \cdots \oplus a_{n} x_{n} \oplus c$, where $a_{j}, c \in G F(2), j=1,2, \ldots, n$, has all the vectors in $V_{n}$ as its linear structures. Hence having linear structures is generally regarded as an unwelcome property in cryptographic practice.

First we can see that the SAC is an indicator with a very strong local flavor, as it guarantees good avalanche characteristics with respect only to the vectors of Hamming weight one. A function that satisfies the SAC can have a large number of vectors of Hamming weight larger than one as its linear structures. Such functions, if employed in certain cryptographic algorithms or systems, can result in a potential security risk.

Next we consider generalizations of the SAC. The higher order SAC suggested by Forré in [For89] has not been widely accepted by the research community as a criterion of cryptographic significance, although the concept itself seems interesting from a combinatorial point of view. In contrast, the other generalization of the SAC, namely the propagation criterion, has well established its position in cryptographic design. This can be seen from work represented by [AT90, PLL+91, PGV91, DT93, SZ794b, SZ795]. A function satisfying the propagation criterion of degree $k$ shows the perfect avalanche characteristic with respect to vectors of Hamming weight not larger than $k$. This property, however, does not rule out the possibility that the function can have vectors of Hamming weight larger than $k$ as its linear structures. For instance, all currently known methods for constructing functions satisfying higher degree propagation criteria, including those presented in [PGV91, DT93, SZZ94b, SZZ95], yield functions having undesirable linear structures. Therefore the propagation criterion, though being an extension of the SAC, is merely another indicator for local properties. On the other hand, the criterion is too strict in the sense that it requires that $f(x) \oplus f(x \oplus \alpha)$ be $100 \%$ balanced. This leads to the situation where a function satisfying the propagation criterion of the largest possible degree becomes bent. Although bent functions have nice properties, they are not balanced and hence can hardly be directly employed in practice.

In designing a cryptographic algorithm, we often need functions that satisfy a number of crucial cryptographic requirements such as balance, high nonlinearity, high algebraic degree and good avalanche characteristics. A function can be considered to have good avalanche characteristics if it does not have a nonzero linear structure and satisfies the propagation criterion with respect to the majority of the vectors.

These discussions show a necessity to search for a new criterion for cryptographic functions. The new criterion should overcome the shortcomings of the SAC or its generalizations, and be able to forecast the overall avalanche characteristic of a cryptographic function. The main aim of this paper is to put forward two closely related indicators that forecast the GAC or global avalanche charac- 
teristic of a cryptographic function. We also present methods for constructing functions that have promising overall avalanche characteristics.

The rest of the paper is organized as follows: The two new indicators, one is called the sum-of-squares indicator and the other the absolute indicator, are introduced in Section 2, and the lower and upper bounds on the two indicators are discussed in Sections 3 and 4 respectively. Finally, Section 5 presents two methods, one for even and the other for odd dimensional spaces, for constructing cryptographic functions that have excellent nonlinear characteristics, including GACs, nonlinearity and balance.

\section{Introducing the GAC}

A function on $V_{n}$ is a mapping from $V_{n}$ into GF(2). The truth table of $f$ is a $(0,1)$-sequence defined by $\left(f\left(\alpha_{0}\right), f\left(\alpha_{1}\right), \ldots, f\left(\alpha_{2^{n}-1}\right)\right)$, where $\alpha_{0}=(0, \ldots, 0,0)$, $\alpha_{1}=(0, \ldots, 0,1), \ldots, \alpha_{2^{n-1}-1}=(1, \ldots, 1,1)$. The sequence of $f$ is a $(1,-1)-$ sequence defined by $\left((-1)^{f\left(\alpha_{0}\right)},(-1)^{f\left(\alpha_{1}\right)}, \ldots,(-1)^{f\left(\alpha_{2}{ }^{n}-1\right)}\right)$, where each exponent is regarded as being real-valued.

$f$ is said to show the perfect avalanche effect with respect to a vector $\alpha \in$ $V_{n}$ if it satisfies the propagation criterion with respect to the vector, namely, $f(x) \oplus f(x \oplus \alpha)$ is balanced. We note that $f(x) \oplus f(x \oplus \alpha)$ is also called the directional derivative of $f$ in the direction $\alpha$. To broaden our observation, we say that $f$ shows good avalanche effect with respect to $\alpha$ if $f(x) \oplus f(x \oplus \alpha)$ is almost balanced. By imposing certain conditions on $\alpha$, we have the notion of the SAC as well as that of the propagation criterion. As shown in the previous section, this approach introduces various limitations in capturing the GAC or global avalanche characteristic of a cryptographic function. To get around the problem, we will not impose restrictions on $\alpha$. Instead, we will let it be a free vector, which allows us to examine the overall avalanche characteristic of a function. The following are a few notations used in further discussions.

Let $\tilde{a}=\left(a_{1}, \cdots, a_{m}\right)$ and $\tilde{b}=\left(b_{1}, \cdots, b_{m}\right)$ be two vectors (or sequences), the scalar product of $\tilde{a}$ and $\tilde{b}$, denoted by $\langle\tilde{a}, \tilde{b}\rangle$, is defined as the sum of the component-wise multiplications. In particular, when $\tilde{a}$ and $\tilde{b}$ are from $V_{m},\langle\tilde{a}, \tilde{b}\rangle=$ $a_{1} b_{1} \oplus \cdots \oplus a_{m} b_{m}$, where the addition and multiplication are over $G F(2)$, and when $\tilde{a}$ and $\tilde{b}$ are $(1,-1)$-sequences, $\langle\tilde{a}, \tilde{b}\rangle=\sum_{i=1}^{m} a_{i} b_{i}$, where the addition and multiplication are over the reals.

Given a function $f$ on $V_{n}$ and a vector $\alpha \in V_{n}$, we denote by $\xi(\alpha)$ the sequence of $f(x \oplus \alpha)$. Note that $\xi(0)$ is identical to the sequence of $f$. In addition, $\xi(0) *$ $\xi(\alpha)$, the component-wise multiplication of the two sequences, is the sequence of $f(x) \oplus f(x \oplus \alpha)$. Set $\Delta_{f}(\alpha)=\langle\xi(0), \xi(\alpha)\rangle$. $\Delta_{f}(\alpha)$ is called the auto-correlation of $f$ with a shift $\alpha$. To further simplify our discussions, $\Delta_{f}(\alpha)$ will be written as $\Delta(\alpha)$ if the function under consideration is clear. Obviously, $\Delta(\alpha)=0$ if and only if $f(x) \oplus f(x \oplus \alpha)$ is balanced, and $|\Delta(\alpha)|=2^{n}$ if and only if $f(x) \oplus f(x \oplus \alpha)$ is a constant, namely, $\alpha$ is a linear structure of $f$. More generally, we have

Lemma 1. Let $f$ be a function on $V_{n}$. Then the Hamming weight of the truth table of $f(x) \oplus f(x \oplus \alpha)$ is equal to $2^{n-1}-\frac{1}{2} \Delta(\alpha)$.

Let $e_{+}$and $e_{-}$denote the number of ones and minus ones in $\xi(0) * \xi(\alpha)$ respectively. Thus $e_{+}-e_{-}=\Delta(\alpha),\left(2^{n}-e_{-}\right)-e_{-}=\Delta(\alpha)$ and hence $e_{-}=$ 
$2^{n-1}-\frac{1}{2} \Delta(\alpha)$. As $e_{-}$is also the number of ones in the truth table of $f(x) \oplus f(x \oplus \alpha)$, the lemma holds.

The overall avalanche characteristic of a function $f$ can be measured by examining $|\Delta(\alpha)|$ for all nonzero vectors $\alpha$. We can say that a function has a good GAC or global avalanche characteristic if for most nonzero $\alpha,|\Delta(\alpha)|$ is zero or very close to zero. Again only bent functions that are unbalanced satisfy the criterion perfectly ! In designing cryptographic algorithms, however, we are mainly interested in balanced functions.

Although simple, the concept of GAC introduces a number of problems to be resolved. These include

1. How to measure precisely the GAC of a function.

2. How to compare the GACs of two different functions.

3. What is the best GAC of a balanced function and how to construct balanced functions that achieve the best GAC.

To solve the various problems, we propose the following two indicators:

Definition 2. Let $f$ be a function on $V_{n}$. Then the sum-of-squares indicator for the avalanche characteristic of $f$ is defined by

$$
\sigma_{f}=\sum_{\alpha \in V_{n}} \Delta^{2}(\alpha)
$$

and the absolute indicator for the characteristic is defined by

$$
\Delta_{f}=\max _{\alpha \in V_{n}, \alpha \neq 0}|\Delta(\alpha)| .
$$

The smaller $\sigma_{f}$ and $\Delta_{f}$, the better the GAC of a function. Like many other nonlinearity characteristics of a function including nonlinearity, algebraic degree and the profile of difference distribution tables, the two indicators for the GAC are invariant under nonsingular linear transforms on the input coordinates.

\section{The Sum-of-Squares Indicator $\sigma_{f}$}

A $(1,-1)$-matrix $H$ of order $m$ is called a Hadamard matrix if $H H^{t}=m I_{m}$, where $H^{t}$ is the transpose of $H$ and $I_{m}$ is the identity matrix of order $m$. A Sylvester-Hadamard matrix of order $2^{n}$, denoted by $H_{n}$, is defined by the following recursive relation

$$
H_{0}=1, H_{n}=\left[\begin{array}{cc}
H_{n-1} & H_{n-1} \\
H_{n-1} & -H_{n-1}
\end{array}\right], n=1,2, \ldots
$$

Let $\ell_{i}$ be the $i$ th row of $H_{n}$. By Lemma 2 of [SZZ94b], $\ell_{i}$ is the sequence of a linear function defined by $\varphi_{i}(x)=\left\langle\alpha_{i}, x\right\rangle=a_{1} x_{1} \oplus a_{2} x_{2} \oplus \cdots \oplus a_{n} x_{n}$, where $x=\left(x_{1}, x_{2}, \ldots, x_{n}\right)$ and $\alpha_{i}=\left(a_{1}, a_{2}, \ldots, a_{n}\right)$ is the $i$ th vector in $V_{n}$ in the ascending alphabetical order. 
Definition 3. Let $f$ be a function on $V_{n}$. The Walsh-Hadamard transform of $f$ is defined as

$$
\hat{f}(\alpha)=2^{-\frac{n}{2}} \sum_{x \in V_{n}}(-1)^{f(x) \oplus\langle\alpha, x\rangle}
$$

where $\alpha=\left(a_{1}, \ldots, a_{n}\right) \in V_{n}, x=\left(x_{1}, \ldots, x_{n}\right)$ and $\langle\alpha, x\rangle=\bigoplus_{i=1}^{n} a_{i} x_{i}$, and $f(x) \oplus\langle\alpha, x\rangle$ is regarded as a real-valued function.

The Walsh-Hadamard transform has numerous applications in areas ranging from physical science to communications engineering. It appears in several slightly different forms [Rot76, MS77, Dil72]. The above definition follows the first formula in [Rot76]. It can be equivalently written as

$$
\left(\hat{f}\left(\alpha_{0}\right), \hat{f}\left(\alpha_{1}\right), \ldots, \hat{f}\left(\alpha_{2^{n}-1}\right)\right)=2^{-\frac{n}{2}} \xi H_{n}
$$

where $\alpha_{i}$ is the $i$ th vector in $V_{n}$ according to the ascending order, $\xi$ is the sequence of $f$ and $H_{n}$ is the Sylvester-Hadamard matrix of order $2^{n}$. More information regarding the transform can be found in [MS77, Dil72]. In addition, Beauchamp's book [Bea84] is a good source of information on other related orthogonal transforms with their applications.

We now introduce the concept of bent functions.

Definition 4. A function $f$ on $V_{n}$ is called a bent function if its Walsh-Hadamard transform satisfies

$$
\hat{f}(\alpha)= \pm 1
$$

for all $\alpha \in V_{n}$.

From [Dil72, AT90, SMZ93, YH89] we know that the following four statements are equivalent

(i) $f$ is bent.

(ii) $\langle\xi, \ell\rangle= \pm 2^{\frac{1}{2} n}$ for any affine sequence $\ell$ of length $2^{n}$, where $\xi$ is the sequence of $f$.

(iii) $f(x) \oplus f(x \oplus \alpha)$ is balanced for any non-zero vector $\alpha \in V_{n}$, where $x=$ $\left(x_{1}, x_{2}, \ldots, x_{n}\right)$.

(iv) $M=\left((-1)^{f\left(\alpha_{i} \oplus \alpha_{j}\right)}\right), 0 \leq i, j \leq 2^{n}-1$, which is called the matrix of $f$ is a Hadamard matrix.

\subsection{Bounds on $\sigma_{f}$}

McFarland, when studying Walsh-Hadamard transform of functions, obtained the following result (see also Theorem 3.3 of [Dil72]):

$$
M=2^{-n} H_{n} \operatorname{diag}\left(\left\langle\xi, \ell_{0}\right\rangle, \cdots,\left\langle\xi, \ell_{2^{n}-1}\right\rangle\right) H_{n},
$$

where $f$ is a function on $V_{n}, \xi$ is the sequence of $f, M=\left((-1)^{f\left(\alpha_{i} \oplus \alpha_{j}\right)}\right), 0 \leq$ $i, j \leq 2^{n}-1$, and $\ell_{i}$ is the $i$ th row of $H_{n}$. Thus

$$
M M^{T}=2^{-n} H_{n} \operatorname{diag}\left(\left\langle\xi, \ell_{0}\right\rangle^{2}, \cdots,\left\langle\xi, \ell_{2^{n}-1}\right\rangle^{2}\right) H_{n}
$$


The first row of $M M^{T}$ is

$$
\left(\Delta\left(\alpha_{0}\right), \Delta\left(\alpha_{1}\right), \cdots, \Delta\left(\alpha_{2^{n}-1}\right)\right)
$$

while the first row of $2^{-n} H_{n} \operatorname{diag}\left(\left\langle\xi, \ell_{0}\right\rangle^{2}, \cdots,\left\langle\xi, \ell_{2^{n}-1}\right\rangle^{2}\right) H_{n}$ can be expressed as

$$
2^{-n}\left(\left\langle\xi^{*}, \ell_{0}\right\rangle, \cdots,\left\langle\xi^{*}, \ell_{2^{n}-1}\right\rangle\right)=2^{-n} \xi^{*} H_{n}
$$

where

$$
\xi^{*}=\left(\left\langle\xi, \ell_{0}\right\rangle^{2}, \cdots,\left\langle\xi, \ell_{2^{n}-1}\right\rangle^{2}\right)
$$

Hence

$$
\left(\Delta\left(\alpha_{0}\right), \Delta\left(\alpha_{1}\right), \cdots, \Delta\left(\alpha_{2^{n}-1}\right)\right)=2^{-n}\left(\left\langle\xi, \ell_{0}\right\rangle^{2}, \cdots,\left\langle\xi, \ell_{2^{n}-1}\right\rangle^{2}\right) H_{n} .
$$

Thus we have proved:

Theorem 5. Let $\xi$ be the sequence of a function $f$ on $V_{n}$. Then

$$
\left(\Delta\left(\alpha_{0}\right), \Delta\left(\alpha_{1}\right), \cdots, \Delta\left(\alpha_{2^{n}-1}\right)\right) H_{n}=\left(\left\langle\xi, \ell_{0}\right\rangle^{2}, \cdots,\left\langle\xi, \ell_{2^{n}-1}\right\rangle^{2}\right) .
$$

This theorem is in fact a special form of a more general result, the WienerKhintchine Theorem [Bea84]. Now write $\eta=\left(\Delta\left(\alpha_{0}\right), \Delta\left(\alpha_{1}\right), \cdots, \Delta\left(\alpha_{2^{n}-1}\right)\right)$. Since

$$
\left\langle\xi^{*}, \xi^{*}\right\rangle=\left\langle\eta H_{n}, \eta H_{n}\right\rangle=\eta H_{n} H_{n}^{T} \eta^{T}=2^{n}\langle\eta, \eta\rangle,
$$

we have

$$
\sum_{j=0}^{2^{n}-1}\left\langle\xi, \ell_{j}\right\rangle^{4}=2^{n} \sum_{\alpha \in V_{n}} \Delta^{2}(\alpha) .
$$

Thus the following result holds:

$$
\sigma_{f}=\sum_{\alpha \in V_{n}} \Delta^{2}(\alpha)=2^{-n} \sum_{j=0}^{2^{n}-1}\left\langle\xi, \ell_{j}\right\rangle^{4}
$$

A closely related equation is

$$
\sum_{j=0}^{2^{n}-1}\left\langle\xi, \ell_{j}\right\rangle^{2}=2^{2 n}
$$

(See also p.416, [MS77]). Both (1) and (2) are special forms of a general equation attributed to Parseval [Bea84].

The nonlinearity of a function $f$ on $V_{n}$, commonly denoted by $N_{f}$, is defined as the minimum Hamming distance between $f$ and all the affine functions on $V_{n}$. On the other hand, the distance between two functions $g_{1}$ and $g_{2}$ on $V_{n}$, namely the number of disagreeing positions in the truth tables or sequences of the two functions, can be calculated by

$$
d\left(g_{1}, g_{2}\right)=2^{n-1}-\frac{1}{2}\left\langle\eta_{1}, \eta_{2}\right\rangle
$$


where $\eta_{i}, i=1,2$, are the sequences of $g_{1}$ and $g_{2}$ (see for instance Lemma 4 of [SZZ94b]). Hence for any $f$ on $V_{n}$, we have

$$
N_{f}=2^{n-1}-\frac{1}{2} \max \left\{\left|\left\langle\xi, \ell_{i}\right\rangle\right|, 0 \leq i \leq 2^{n}-1\right\}
$$

where $\xi$ is the sequence of $f$ and $\ell_{0}, \ldots, \ell_{2^{n}-1}$ are the rows of $H_{n}$, namely, the sequences of the linear functions on $V_{n}$. Now considering Theorem 5, Lemma 1 and in particular, the equation (1), we can see that the nonlinearity of a function is closely related to the sum-of-squares avalanche characteristic of the function. In general, the larger the nonlinearity, the smaller (i.e., better) the sum-of-squares avalanche characteristic.

Theorem 6. Let $f$ be a function on $V_{n}$. Then

(i) $2^{2 n} \leq \sigma_{f} \leq 2^{3 n}$,

(ii) $\sigma_{f}=2^{2 n}$ if and only if $f$ is a bent function,

(iii) $\sigma_{f}=2^{3 n}$ if and only if $f$ is an affine function.

Proof. (i) Note that $\Delta(0)=2^{n}$. Hence

$$
\sigma_{f}=\sum_{\alpha \in V_{n}} \Delta^{2}(\alpha) \geq \Delta^{2}(0)=2^{2 n} .
$$

On the other hand, by Parseval's equation (2), we have

$$
\sum_{j=0}^{2^{n}-1}\left\langle\xi, \ell_{j}\right\rangle^{2}=2^{2 n}
$$

Thus

$$
\sigma_{f}=2^{-n} \sum_{j=0}^{2^{n}-1}\left\langle\xi, \ell_{j}\right\rangle^{4} \leq 2^{-n}\left(\sum_{j=0}^{2^{n}-1}\left\langle\xi, \ell_{j}\right\rangle^{2}\right)^{2}=2^{-n} 2^{4 n}=2^{3 n} .
$$

(ii) $\sigma_{f}=2^{2 n}$ if and only if $\Delta(\alpha)=0$ for all $\alpha \neq 0$, namely, $f$ is bent.

(iii) Set $b_{j}=\left\langle\xi, \ell_{j}\right\rangle^{2}$. Again by Parseval's equation (2), $\sum_{j=0}^{2^{n}-1} b_{j}=2^{n}$. Now we have the following reasoning:

$\sigma_{f}=2^{3 n}$ if and only if

$2^{-n} \sum_{j=0}^{2^{n}-1} b_{j}^{2}=2^{3 n}$ if and only if

$\sum_{j=0}^{2^{n}-1} b_{j}^{2}=2^{4 n}$ if and only if

$\sum_{j=0}^{2^{n}-1} b_{j}^{2}=\left(\sum_{j=0}^{2^{n}-1} b_{j}\right)^{2}$ if and only if

$b_{i} b_{j}=0$ for $j \neq i$ if and only if

there exists a $j_{0}$ such that $b_{j_{0}}=2^{2 n}$ and $b_{j}=0$ for $j \neq j_{0}$ if and only if

there exists a $j_{0}$ such that $\left\langle\xi, \ell_{j_{0}}\right\rangle= \pm 2^{n}$ and $\left\langle\xi, \ell_{j}\right\rangle=0$ for $j \neq j_{0}$ if and only if there exists a $j_{0}$ such that $\xi= \pm \ell_{j_{0}}$, i.e., $f$ is an affine function.

A more important topic is to find a lower bound on $\sigma_{f}$ for balanced functions $f$. This is left as a problem for future research. 


\section{2 $\sigma_{f}$ of Some Highly Nonlinear Functions}

Now we discuss the sum-of-squares avalanche characteristics of some highly nonlinear functions.

The structure of a function $f$ on $V_{n}$ that satisfies the propagation criterion with respect to all but a subset $\Re$ of vectors in $V_{n}$, has been studied in [SZZ94d]. We note that $\Re$ always contains the zero vector in $V_{n}$. It has been shown in [SZZ94d] that

1. if $|\Re|=2$ then $n$ is odd, the nonlinearity of $f$ satisfies $N_{f}=2^{n-1}-2^{\frac{1}{2}(n-1)}$ and in addition, there exists a nonsingular matrix of order $n$ over GF(2), say $A$, such that $g(x)=f(A x)$ can be written as

$$
g(x)=c x_{n} \oplus h\left(x_{1}, \ldots, x_{n-1}\right)
$$

where $c$ is a constant in $\mathrm{GF}(2)$ and $h$ is a bent function on $V_{n-1}$;

2. if $|\Re|=4$ then $n$ must be even, the nonlinearity of $f$ satisfies $N_{f}=2^{n-1}-2^{\frac{1}{2} n}$ and there exists a nonsingular matrix of order $n$ over GF(2), say $B$, such that $g(x)=f(B x)$ can be written as

$$
g(x)=c_{1} x_{n-1} \oplus c_{2} x_{n} \oplus h\left(x_{1}, \ldots, x_{n-2}\right)
$$

where $c_{1}$ and $c_{2}$ are constants in $\mathrm{GF}(2)$, and $h$ is a bent function on $V_{n-2}$; 3 . in both cases, all vectors in $\Re$ are linear structures of $f$.

Now the sum-of-squares avalanche characteristics for the two cases can be determined.

1. if $|\Re|=2$ then $n=2 k+1$ and

$$
\sigma_{f}=\sum_{\alpha \in V_{2 k+1}} \Delta^{2}(\alpha)=\Delta^{2}(0)+\Delta^{2}\left(\alpha_{1}\right)=2 \cdot 2^{4 k+2}=2^{4 k+3}
$$

where $\alpha_{1}$ is the nonzero vector in $\Re$;

2. if $|\Re|=4$ then $n=2 k$ and

$$
\sigma_{f}=\sum_{\alpha \in V_{2 k}} \Delta^{2}(\alpha)=\Delta^{2}(0)+\sum_{j=1}^{3} \Delta^{2}\left(\alpha_{j}\right)=4 \cdot 2^{4 k}=2^{4 k+2}
$$

where $\alpha_{i}, i=1,2,3$, are the nonzero vectors in $\Re$.

Functions $f$ on $V_{n}$ with $|\Re|=5$ are also studied in [SZZ94d], where it is shown that $N_{f}=2^{n-1}-2^{\frac{1}{2}(n-1)}, n$ is odd and that $\left|\Delta\left(\alpha_{i}\right)\right|=2^{n-1}$ for all the four nonzero vectors, $\alpha_{1}, \alpha_{1}, \alpha_{3}$ and $\alpha_{4}$, in the set $\Re$. Thus, the sum-of-squares avalanche characteristic of $f$ with $\Re=5$ is

$$
\sigma_{f}=\sum_{\alpha \in V_{2 k+1}} \Delta^{2}(\alpha)=\Delta^{2}(0)+\sum_{j=1}^{4} \Delta^{2}\left(\alpha_{j}\right)=2^{4 k+2}+4 \cdot 2^{4 k}=2^{4 k+3} .
$$

This value is the same as that for the case when $|\Re|=2$.

It is also shown in [SZZ94d] that functions with $|\Re|=3$ or 6 do not exist. 


\section{The Absolute Indicator $\Delta_{f}$}

Let $f$ be a function on $V_{n}$. Recall that $\Delta_{f}$ is defined as the maximum among all $\Delta(\alpha), \alpha \neq 0$, and that $\Delta(\alpha)= \pm 2^{n}$ if and only if $\alpha$ is a linear structure of $f$. Thus the following result is straightforward.

Lemma 7. Let $f$ be a function on $V_{n}$. Then $0 \leq \Delta_{f} \leq 2^{n}$. Moreover, $\Delta_{f}=0$ if and only if $f$ is bent, and $\Delta_{f}=2^{n}$ if and only if $f$ has a nonzero linear structure.

In particular, for any quadratic non-bent function $f$, we have $\Delta_{f}=2^{n}$. Next we focus on functions whose algebraic degrees are at least three.

Now set $g(x)=f(x) \oplus f(x \oplus \alpha)$. Then the algebraic degree of $g$ is one less than that of $f$. As $g(x) \oplus g(x \oplus \alpha)=0, g$ cannot be bent. Thus we have the following simple yet helpful lemma.

Lemma 8. Let $f$ be a function on $V_{n}$. Then for any nonzero vector $\alpha \in V_{n}$, $f(x) \oplus f(x \oplus \alpha)$ is not bent and its algebraic degree is one less than that of $f$.

Recall that by Lemma $1, \Delta_{f}(\alpha)$ and the Hamming weight $W(g)$ of $g(x)=$ $f(x) \oplus f(x \oplus \alpha)$ are related by $W(g)=2^{n-1}-\frac{1}{2} \Delta_{f}(\alpha)$, or equivalently, $\Delta_{f}(\alpha)=$ $2\left(2^{n-1}-W(g)\right)$. Therefore, assume that $f$ is a function on $V_{n}$ of algebraic degree $k$, the problem of finding $\Delta_{f}$ is reduced to that of finding the minimum Hamming weight of functions on $V_{n}$ of algebraic degree $k-1$ which are integrable in the sense that they can be expressed as $f(x) \oplus f(x \oplus \alpha)$ with $\alpha$ a nonzero vector in $V_{n}$.

For a function $f$ on $V_{n}$ of algebraic degree $k \geq 3, \Delta_{f}$ is to some extent connected to the weight distribution of the $(k-1)$ st order binary Reed-Muller code $\operatorname{RM}(k-1, n)$. Here $\operatorname{RM}(r, n)$ is defined as the collection of all functions on $V_{n}$, whose algebraic degrees are at most $r$. The minimum Hamming weight of $\operatorname{RM}(r, n)$, i.e., the minimum Hamming weight of functions on $V_{n}$ of algebraic degree $r$, is known to be $2^{n-r}$ (see Theorem 3, p.375 of [MS77]). Now the connection between $\Delta_{f}$ of a function $f$ on $V_{n}$ of algebraic degree $k$ and $\operatorname{RM}(k-1, n)$ can be precisely stated as

$$
\Delta_{f} \geq 2\left(2^{n-1}-2^{n-k+1}\right)=2^{n}-2^{n-k+2}
$$

where $2^{n-k+1}$ is the minimum Hamming weight of $\operatorname{RM}(k-1, n)$. This lower bound on $\Delta_{f}$, however, is very rough and not satisfactory. The reason is that $2^{n-k+1}$ is the minimum Hamming weight of all functions on $V_{n}$, whose algebraic degrees are $k-1$, including those which are not integrable. Hence it is one of our aims to find a lower bound on $\Delta_{f}$ that is smaller (i.e., better) than the value $2^{n}-2^{n-k+2}$.

On the other hand, in designing cryptographic algorithms we are more concerned with balanced nonlinear functions than non-balanced ones. Therefore it is an important issue to know how small the absolute indicator $\Delta_{f}$ can be, for a balanced nonlinear function $f$ on $V_{n}$. In the rest of the section we report the result we have obtained on the lower bound of $\Delta_{f}$ of cubic functions. This result can be regarded as the first step towards fully answering the question about $\Delta_{f}$.

The following two results (see for instance Lemma 9 of [SMZ93] and Lemma 5 of [SZZ94c] respectively), will be employed in the discussions of cubic functions. 
Lemma 9. $f\left(x_{1}, \ldots, x_{n}\right)=\psi\left(x_{1}, \ldots, x_{r}\right) \oplus h\left(x_{r+1}, \ldots, x_{n}\right)$ is balanced on $V_{n}$ if $\psi$ is balanced on $V_{r}$ or $h$ is balanced on $V_{n-r}$.

Lemma 10. If $f$ is a quadratic function and does not have nonzero linear structures, then it is bent.

According to Lemma 10, a quadratic non-bent function $f$ must have at least one linear structure. Hence the lower bound on $\Delta_{f}$ for such a function is (trivially) equal to $2^{n}$. For cubic functions, we have a result described in the following theorem.

Theorem 11. Let $f$ be a non-bent cubic function on $V_{n}$. Then $\Delta_{f} \geq 2^{\frac{1}{2}(n+1)}$.

Proof. Since $f$ is not bent, there exists a nonzero vector in $V_{n}$, say $\alpha$, such that $f(x) \oplus f(x \oplus \alpha)$ is not balanced. We set $g(x)=f(x) \oplus f(x \oplus \alpha)$ and want to find out the Hamming weight of the truth table of $g$ from which we can find out $\Delta(\alpha)$ and hence the lower bound on $\Delta_{f}$.

By Lemma $8, g$ is not bent. Note that $g$ is quadratic. By Lemma $10, g$ has nonzero linear structures. it is easy to see [Nyb93] that all the linear structures of a function on $V_{n}$ form a linear subspace of $V_{n}$. Denote by $W$ the linear subspace formed by the linear structures of $g$, and by $r$ the dimension of $W$. From [SZZ94c], there exists a nonsingular matrix $A$ of order $n$ on $\mathrm{GF}(2)$ such that $g^{*}(x)=g(x A)$ can be expressed as

$$
g^{*}\left(x_{1}, \ldots, x_{n}\right)=\psi\left(x_{1}, \ldots, x_{r}\right) \oplus h\left(x_{r+1}, \ldots, x_{n}\right)
$$

where $\psi$ is a linear function on $W$ while $h$ is a function on $V_{n-r}$ that does not have nonzero linear structures. Note that the truth tables of $g^{*}$ and $g$ have the same Hamming weight. Now suppose that $\psi$ is a nonzero linear function. Then $\psi$ is balanced. By Lemma $9, g^{*}$ is balanced, which contradicts the fact that $g$ is not balanced. Consequently $\psi$ must be equal to zero and hence

$$
g\left(x_{1}, \ldots, x_{n}\right)=h\left(x_{r+1}, \ldots, x_{n}\right) .
$$

As $h$ does not have nonzero linear structures, by Lemma 10, it is a bent function on $V_{n-r}$ (which implies that $n-r$ must be even). Thus the Hamming weight of the truth table of $h$ is $2^{n-r-1}+c 2^{\frac{1}{2}(n-r)-1}$, where $c= \pm 1$, and the Hamming weight of the truth table of $g^{*}$, a function on $V_{n}$, is $2^{r}\left(2^{n-r-1}+\right.$ $\left.c 2^{\frac{1}{2}(n-r)-1}\right)=2^{n-1}+c 2^{\frac{1}{2}(n+r)-1}$. Equivalently, the Hamming weight of the truth table of $f(x) \oplus f(x \oplus \alpha)$ is $2^{n-1}+c 2^{\frac{1}{2}(n+r)-1}$. Applying Lemma 1 to the function $f$, we have $\Delta(\alpha)=c 2^{\frac{1}{2}(n+r)}$. Thus we have proved that there exists a nonzero vector $\alpha \in V_{n}$ such that $|\Delta(\alpha)|=2^{\frac{1}{2}(n+r)}$. As $r$, the dimension of $W$, is at least 1 , we have $\Delta_{f} \geq|\Delta(\alpha)| \geq 2^{\frac{1}{2}(n+1)}$.

We stress that the bound $2^{\frac{1}{2}(n+1)}$ in Theorem 11 is satisfied by any non-bent cubic function, be it balanced or non-balanced. The bound, however, is clearly not satisfied by functions of algebraic degree larger than three. For instance, complementing a single bit in the truth table of a bent function $f$ on $V_{n}$ results in a non-bent, non-balanced function $g$ with $\Delta_{g}(\alpha)= \pm 2$ for all nonzero $\alpha \in V_{n}$ (hence $\Delta_{g}=2$, and by Theorem 11, $g$ can not be cubic.) Nevertheless, we believe that the lower bound $2^{\frac{1}{2}(n+1)}$ is also satisfied by balanced functions of algebraic degree larger than three. This leads to the following conjecture: 
Conjecture 1 Let $f$ be a balanced function on $V_{n}$, whose algebraic degree is at least three. Then $\Delta_{f} \geq 2^{\frac{1}{2}(n+1)}$.

\section{Constructing Balanced Functions with Good GAC}

Having discussed various bounds of the two indicators $\sigma_{f}$ and $\Delta_{f}$, we now turn our attention to constructing cryptographic functions that have good GACs or global avalanche characteristics measured in terms of the two indicators. A remarkable property of the functions to be constructed is that they are balanced and do not have a nonzero linear structure.

\subsection{On $V_{2 k}$}

For $z \in V_{2 k}$, write $z=(y, x)$ where $y \in V_{k}$ and $x \in V_{k}$. Let $\omega$ be a permutation on the set of nonzero vectors in $V_{k}$, i.e., $V_{k}-\{0\}=\left\{\alpha_{1}, \ldots, \alpha_{2^{k}-1}\right\}$, where $\alpha_{j}$ is the $i$ th vector in $V_{k}$ in the ascending alphabetical order. Set

$$
f(z)=f(y, x)= \begin{cases}\left\langle\alpha_{j_{0}}, x\right\rangle & \text { if } y=0 \\ \langle\omega(y), x\rangle & \text { if } y \neq 0\end{cases}
$$

where $\langle\cdot, \cdot\rangle$ denotes the scalar product and $\alpha_{j_{0}}$ is a fixed nonzero vector in $V_{k}$. Equivalently (5) can be expressed as

$f(z)=\left(1 \oplus y_{1}\right)\left(1 \oplus y_{2}\right) \cdots\left(1 \oplus y_{k}\right)\left\langle\alpha_{j_{0}}, x\right\rangle \oplus\left[1 \oplus\left(1 \oplus y_{1}\right)\left(1 \oplus y_{2}\right) \cdots\left(1 \oplus y_{k}\right)\right]\langle\omega(y), x\rangle$

where $y=\left(y_{1}, y_{2}, \ldots, y_{k}\right)$.

First we examine the sequence of the function $f$. Given a vector $\alpha_{i} \in V_{k}$, denote by $\ell_{i}$ the sequence of a linear function on $V_{k}$ defined by $\left\langle\alpha_{i}, x\right\rangle$. By Lemma 2 of [SMZ93], $\ell_{i}$ is the $i$ th row of $H_{k}, i=0,1, \ldots, 2^{k}-1$. Since $\alpha_{j}$ corresponds to the binary representation of integer $j, w$ can be regarded as a permutation on $\left\{1, \ldots, 2^{k}-1\right\}$. In particular, $\omega\left(\alpha_{j}\right)=\alpha_{i}$ can be equivalently written as $\omega(j)=i$. By Lemma 1 of [SMZ93], the sequence of $f$ defined by (5) is

$$
\xi=\left(\ell_{j_{0}}, \ell_{\omega(1)}, \ldots, \ell_{\omega\left(2^{k}-1\right)}\right)
$$

We can view $\xi$ in the following way: Concatenating the rows in $H_{k}$ together, we have $\left(\ell_{0}, \ell_{1}, \ldots, \ell_{2^{k}-1}\right)$. Replacing $\ell_{0}$ by $\ell_{j_{0}}$ gives us $\left(\ell_{j_{0}}, \ell_{1}, \ell_{2}, \ldots, \ell_{2^{k}-1}\right)$. Finally reordering $\ell_{1}, \ldots, \ell_{2^{k}-1}$ according to the permutation $\omega$ results in the sequence $\xi$. As each $\ell_{i}, 1 \leq i \leq 2^{k}-1$, contains an equal number of ones and minus ones, their concatenation $\xi$ has the same property. Thus we have

Lemma 12. $f$ defined by (5) is a balanced function on $V_{2 k}$.

We proceed to the discussion of the absolute indicator $\Delta_{f}$. Let $\gamma=(\beta, \alpha)$ be a nonzero vector in $V_{2 k}$, where $\beta, \alpha \in V_{k}$. By definition,

$$
\Delta(\gamma)=\sum_{y \in V_{k}} \sum_{x \in V_{k}}(-1)^{f(y, x) \oplus f(y \oplus \beta, x \oplus \alpha)} .
$$

We discuss $\Delta(\gamma)$ in two separate cases: $\beta \neq 0$ and $\beta=0$. 
First we consider Case 1 where $\beta \neq 0$. In this case $\Delta(\gamma)$ can be written as

$$
\Delta(\gamma)=\sum_{y=0, \beta} \sum_{x \in V_{k}}(-1)^{f(y, x) \oplus f(y \oplus \beta, x \oplus \alpha)}+\sum_{y \neq 0, \beta} \sum_{x \in V_{k}}(-1)^{f(y, x) \oplus f(y \oplus \beta, x \oplus \alpha)} .
$$

When $y=0$, the exponent $f(y, x) \oplus f(y \oplus \beta, x \oplus \alpha)$ becomes

$$
f(0, x) \oplus f(\beta, x \oplus \alpha)=\left\langle\alpha_{j_{0}}, x\right\rangle \oplus\langle\omega(\beta), x \oplus \alpha\rangle=\left\langle\alpha_{j_{0}} \oplus \omega(\beta), x\right\rangle \oplus\langle\omega(\beta), \alpha\rangle(6)
$$

and when $y=\beta$, it becomes

$$
f(\beta, x) \oplus f(0, x \oplus \alpha)=\langle\omega(\beta), x\rangle \oplus\left\langle\alpha_{j_{0}}, x \oplus \alpha\right\rangle=\left\langle\alpha_{j_{0}} \oplus \omega(\beta), x\right\rangle \oplus\left\langle\alpha_{j_{0}}, \alpha\right\rangle(7)
$$

Otherwise when $y \neq 0$ or $\beta$, the exponent becomes

$$
\begin{aligned}
f(y, x) \oplus f(y \oplus \beta, x \oplus \alpha) & =\langle\omega(y), x\rangle \oplus\langle\omega(y \oplus \beta), x \oplus \alpha\rangle \\
& =\langle\omega(y) \oplus \omega(y \oplus \beta), x\rangle \oplus\langle\omega(y \oplus \beta), \alpha\rangle .
\end{aligned}
$$

To find out the value of $\Delta(\gamma)$, we distinguish between the cases of $\omega(\beta)=\alpha_{j_{0}}$ and $\omega(\beta) \neq \alpha_{j_{0}}$.

When $\omega(\beta)=\alpha_{j_{0}},(6)$ becomes a constant $\langle\omega(\beta), \alpha\rangle$, (7) also becomes a constant $\left\langle\alpha_{j_{0}}, \alpha\right\rangle$ and (9) is a nonzero linear function of $x$ for any fixed $y$ and hence balanced. Thus we have

$$
\left.\Delta(\gamma)=\sum_{x \in V_{k}}\left[(-1)^{\left\langle\alpha_{j_{0}}, \alpha\right.}\right\rangle+(-1)^{\left\langle\alpha_{j_{0}}, \alpha\right\rangle}\right]=2 \cdot 2^{k} \cdot c=2^{k+1} c
$$

where $c=(-1)^{\left\langle\alpha_{j_{0}}, \alpha\right\rangle}= \pm 1$.

On the other hand, when $\omega(\beta) \neq \alpha_{j_{0}},(6),(7)$ and (9) are all nonzero linear functions and hence balanced. This results in $\Delta(\gamma)=0$.

Next we consider Case 2 where $\beta=0$. In this case, it is necessary for $\alpha$ to be nonzero. Thus (5) specializes to

$$
\Delta(\gamma)=\sum_{x \in V_{k}}(-1)^{f(0, x) \oplus f(0, x \oplus \alpha)}+\sum_{y \neq 0} \sum_{x \in V_{k}}(-1)^{f(y, x) \oplus f(y, x \oplus \alpha) .}
$$

When $y=0$, the exponent $f(y, x) \oplus f(y, x \oplus \alpha)$ becomes

$$
f(0, x) \oplus f(0, x \oplus \alpha)=\left\langle\alpha_{j_{0}}, x\right\rangle \oplus\left\langle\alpha_{j_{0}}, x \oplus \alpha\right\rangle=\left\langle\alpha_{j_{0}}, \alpha\right\rangle .
$$

Otherwise, when $y \neq 0$, it becomes

$$
\begin{aligned}
f(y, x) \oplus f(y, x \oplus \alpha) & =\langle\omega(y), x\rangle \oplus\langle\omega(y), x \oplus \alpha\rangle \\
& =\langle\omega(y), \alpha\rangle .
\end{aligned}
$$

Now $\Delta(\gamma)$ can be calculated by

$$
\begin{aligned}
\Delta(\gamma) & =\sum_{x \in V_{k}}(-1)^{\left\langle\alpha_{j_{0}}, \alpha\right\rangle}+\sum_{y \neq 0} \sum_{x \in V_{k}}(-1)^{\langle\omega(y), \alpha\rangle} \\
& =\sum_{x \in V_{k}}(-1)^{\left\langle\alpha_{j_{0}}, \alpha\right\rangle}+\sum_{u \neq 0} \sum_{x \in V_{k}}(-1)^{\langle u, \alpha\rangle}
\end{aligned}
$$


where $u=\omega(y)$. Since $\omega$ is a permutation on $V_{k}-\{0\}, u=\omega(y) \neq 0$. Thus we can continue our calculation of $\Delta(\gamma)$ :

$$
\Delta(\gamma)=\sum_{x \in V_{k}}(-1)^{\left\langle\alpha_{j_{0}}, \alpha\right\rangle}+\sum_{v \in V_{k}} \sum_{x \in V_{k}}(-1)^{\langle v, \alpha\rangle}-\sum_{x \in V_{k}}(-1)^{\langle 0, \alpha\rangle} .
$$

Note that $\langle v, \alpha\rangle$ is a nonzero linear function of $v$ and hence balanced. Thus we have

$$
\begin{aligned}
\Delta(\gamma) & =\sum_{x \in V_{k}}(-1)^{\left\langle\alpha_{j_{0}}, \alpha\right\rangle}-\sum_{x \in V_{k}}(-1)^{\langle 0, \alpha\rangle} \\
& =\sum_{x \in V_{k}}\left[(-1)^{\left\langle\alpha_{j_{0}}, \alpha\right\rangle}-1\right] \\
& = \begin{cases}0 & \text { if }\left\langle\alpha_{j_{0}}, \alpha\right\rangle=0 \\
2^{k+1} & \text { if }\left\langle\alpha_{j_{0}}, \alpha\right\rangle=1\end{cases}
\end{aligned}
$$

Summarizing the above discussions on Cases 1 and 2, we conclude that $|\Delta(\gamma)| \leq 2^{k+1}$ for any nonzero vector $\gamma \in V_{2 k}$. This proves the following lemma:

Lemma 13. Let $f$ be the function on $V_{2 k}$ defined by (5). Then $\Delta_{f} \leq 2^{k+1}$.

Now we count the vectors with respect to which the function $f$ satisfies the propagation criterion. We have seen in the above discussions that $\Delta(\gamma)=0$ in two cases: (1) $\Delta(\gamma)=0, \beta \neq 0, \omega(\beta) \neq$ or $\alpha_{j_{0}}$ and $\alpha$ is arbitrary. (2) $\Delta(\gamma)=0$, $\beta=0$ and $\alpha$ satisfies $\alpha \neq 0$ and $\left\langle\alpha_{j_{0}}, \alpha\right\rangle=0$. For the first case there are $\left(2^{k}-2\right) 2^{k}=2^{2 k}-2^{k+1}$ choices, while for the second case there are $2^{k-1}-1$ choices for $\gamma=(\beta, \alpha)$. Hence there exist $2^{2 k}-2^{k+1}+2^{k-1}-1$ vectors $\gamma=(\beta, \alpha)$ such that $\Delta(\gamma)=0$. This proves

Lemma 14. The function $f$ defined by (5) satisfies the propagation criterion with respect to $2^{2 k}-2^{k+1}+2^{k-1}-1$ vectors in $V_{2 k}$.

Next we examine the sum-of-squares avalanche characteristic of the function $f$. Recall that the sequence of $f$ is

$$
\xi=\left(\ell_{j_{0}}, \ell_{\omega(1)}, \ell_{\omega(2)}, \ldots, \ell_{\omega\left(2^{k}-1\right)}\right)
$$

where $\ell_{i}$ is the sequence of a linear function on $V_{k}$ defined by $\left\langle\alpha_{i}, x\right\rangle$.

Let $L$ be a row of $H_{2 k}$. By Lemma 2 of [SMZ93], $L$ is a linear sequence of length $2^{2 k}$. Since $H_{2 k}=H_{k} \times H_{k}, L$ can be rewritten as $L=\ell_{p} \times \ell_{q}$ for some $p$ and $q$ satisfying $0 \leq p, q, \leq 2^{k}-1$. Write $\ell_{p}=\left(c_{0}, c_{1}, \ldots, c_{2^{k}-1}\right)$. Then we have $L=\left(c_{0} \ell_{q}, c_{1} \ell_{q}, \ldots, c_{2^{k}-1} \ell_{q}\right)$.

As $H_{k}$ is a Hadamard matrix, $\left\langle\ell_{i}, \ell_{j}\right\rangle=0$ when $j \neq i$. Also note that as $\omega$ is a permutation on $V_{k}-\{0\}, \omega\left(\alpha_{j}\right)$ runs through the nonzero vectors in $V_{k}$ while $j$ runs through $1,2, \ldots, 2^{k}-1$. So there exists a unique $j^{*}$ such that $\omega\left(\alpha_{j^{*}}\right)=\alpha_{j_{0}}$. Thus we have

$$
\langle\xi, L\rangle= \begin{cases}\left.\left(c_{0}+c_{j^{*}}\right)\left\langle\ell_{j_{0}}, \ell_{j_{0}}\right)\right\rangle=\left(c_{0}+c_{j^{*}}\right) 2^{k} & \text { if } \alpha_{q}=\alpha_{j_{0}} \\ \pm 2^{k} & \text { if } \alpha_{q} \neq \alpha_{j_{0}}, 0 \\ 0 & \text { if } q=0\end{cases}
$$


Here $c_{0}=1$ and $c_{j^{*}}= \pm 1$.

There exist $2^{k-1}$ linear sequences $\ell_{p}$ such that $c_{1}=1$. Hence there exist $2^{k-1}$ linear sequences $L$ such that $L=\ell_{p} \times \ell_{q}$ with $c_{j}{ }^{*}=1$ and $\alpha_{q}=\alpha_{j_{0}}$. For these sequences we have $\langle\xi, L\rangle=2^{k+1}$.

For $c_{j^{*}}=-1$, we have $\langle\xi, L\rangle=0$. It is easy to see that there exits $2^{k} \cdot\left(2^{k}-2\right)$ linear sequences $L$ such that $L=\ell_{p} \times \ell_{q}$ with $\alpha_{q} \neq 0$ or $\alpha_{j_{0}}$. With these sequences we have $\langle\xi, L\rangle= \pm 2^{k}$.

In summary, we have

$$
\begin{aligned}
\sigma_{f} & =2^{-2 k} \sum_{s=0}^{2^{2 k}-1}\left\langle\xi, L_{s}\right\rangle^{4}=2^{k-1} \cdot 2^{4(k+1)}+2^{k} \cdot\left(2^{k}-2\right) \cdot 2^{4 k} \\
& =2^{4 k}+2^{3 k+3}-2^{3 k+1} .
\end{aligned}
$$

This proves the following conclusion:

Lemma 15. The sum-of-squares avalanche characteristic of $f$, a function on $V_{2 k}$ defined by (5), satisfies $\sigma_{f}=2^{4 k}+2^{3 k+3}-2^{3 k+1}$.

Recall that for a function on $V_{2 k}$, its sum-of-squares indicator is bounded between $2^{4 k}$ and $2^{6 k}$, with the lower bound $2^{4 k}$ being achieved only when the function is bent. We conjecture that the function $f$ defined by (5) with $\sigma_{f}=2^{4 k}+$ $2^{3 k+3}-2^{3 k+1}$ achieves nearly optimal sum-of-squares avalanche characteristic of balanced functions on $V_{2 k}$.

From the above discussions, it becomes clear that $\left|\left\langle\xi, L_{s}\right\rangle\right|\left\langle 2^{k+1}\right.$ for any $L_{s}$ that is a linear sequence of length $2^{2 k}$. By Lemma 3 of [SMZ93], the nonlinearity of $f$ satisfies $N_{f} \geq 2^{2 k-1}-2^{k}$.

Putting the above discussions together, we have

Theorem 16. Let $f$ be the function on $V_{2 k}$ defined by (5). Then

(i) $f$ is balanced,

(ii) the nonlinearity of $f$ satisfies $N_{f} \geq 2^{2 k-1}-2^{2 k}$,

(iii) $f$ satisfies the propagation criterion with respect to $2^{2 k}-2^{k+1}+2^{k-1}-1$ nonzero vectors,

(iv) the sum-of-squares avalanche characteristic of $f$ satisfies $\sigma_{f}=2^{4 k}+2^{3 k+3}$ $2^{3 k+1}$

(v) the absolute avalanche characteristic of $f$ satisfies $\Delta_{f} \leq 2^{k+1}$.

A final remark is about the strict avalanche characteristic of the function $f$. The number of vectors with respect to which $f$ satisfies the propagation criterion is $2^{2 k}-2^{k+1}+2^{k-1}-1$ which is larger than $2^{2 k-1}$. Hence these vectors contain at least $2 k$ linear independent ones. Let $A$ be the matrix with the $2 k$ linear independent vectors as its rows. Then $A$ is nonsingular and of order $2 k$. By Theorem 3 of [SZZ94a], $f(z A)$ satisfies the SAC. All the properties described in Theorem 16 are affected by the nonsingular transform $A$. 


\subsection{On $V_{2 k+1}$}

To construct functions on $V_{2 k+1}$ with good avalanche characteristics, we need a permutation $m(u)$ on $V_{k}$ with a special property that $u \oplus m(u)$ is also a permutation on $V_{k}$, namely, $u \oplus m(u)$ runs through the vectors in $V_{k}$ while $u$ runs $V_{k}$. As is shown in the following, such functions can be obtained from maximal length shift register sequences or $m$-sequences [Gol82]. In a different context, Nyberg showed that $m$-sequences are useful in constructing cryptographic substitution boxes with the maximum nonlinearity [Nyb91]. (It should be noted, however, that such substitution boxes have been identified to be prone to the differential cryptanalytic attack [BS93, BKPS93].)

Let $\left(s_{0}, s_{1}, \ldots, s_{2^{k}-2}\right)$ be a $m$-sequence of length $2^{k}-1$, where each $s_{i}$ is from GF(2). A $k$-gram is one of the $2^{k}-1$ subsequences of length $k$ of the form

$$
r_{t}=\left(s_{t \bmod \left(2^{k}-1\right)}, s_{(t+1) \bmod \left(2^{k}-1\right)}, \ldots, s_{(t+k-1) \bmod \left(2^{k}-1\right)}\right),
$$

where $t=0,1,2, \ldots, 2^{k}-2$. Note that a $k$-gram can also be viewed as a vector in $V_{k}$. Thus we have an ordered list of $2^{k}-1$ nonzero vectors in $V_{k}\left(r_{0}, r_{1}, \ldots, r_{2^{k}-2}\right)$. Adding to the beginning of the list the zero vector 0 in $V_{k}$ results in an extended ordered list $\left(0, r_{0}, r_{1}, \ldots, r_{2^{k}-2}\right)$. The extended list contains all vectors in $V_{k}$. Rotating cyclically to the left the nonzero vectors in the list by one position we get $\left(0, r_{1}, r_{2}, \ldots, r_{2^{k}-2}, r_{0}\right)$. Now we have two ordered vector lists:

$$
\left(0, r_{0}, r_{1}, \ldots, r_{2^{k}-2}\right)
$$

and

$$
\left(0, r_{1}, r_{2}, \ldots, r_{2^{k}-2}, r_{0}\right) .
$$

Define a mapping $m(u)$ that maps the $i$ th vector in the first list to the corresponding vector in the second list, namely, 0 to $0, r_{0}$ to $r_{1}, r_{1}$ to $r_{2}, \ldots$, and $r_{2^{k}-2}$ to $r_{0}$. By properties of $m$-sequences, the mapping $m(u)$ is a permutation with the special property that $u \oplus m(u)$ is also a permutation.

Now write $W_{1}=\left\{(0, u) \mid u \in V_{k}\right\}, W_{2}=\left\{(1, u) \mid u \in V_{k}\right\}$, where $0,1 \in G F(2)$. Obviously, $V_{k+1}=W_{1} \cup W_{2}$. For any $y \in V_{k+1}$, write $y=\left(y_{1}, u\right)$ where $y_{1} \in$ $G F(2)$ and $u \in V_{k}$. For $z \in V_{2 k+1}$, write $z=(y, x)$ where $y \in V_{k+1}$ and $x \in V_{k}$.

Then the following is our construction for the case of $V_{2 k+1}$ :

$$
f(z)=f(y, x)=\left\{\begin{array}{l}
1 \oplus\langle u, x\rangle \text { if } y \in W_{1} \\
\langle m(u), x\rangle \text { if } y \in W_{2}
\end{array}\right.
$$

where $m(u)$ is a permutation on $V_{k}$ with the property that $u \oplus m(u)$ is also a permutation on $V_{k}$. Note that $(13)$ can be equivalently written as $f(z)=(1 \oplus$ $\left.y_{1}\right)\langle u, x\rangle \oplus y_{1}\langle m(u), x\rangle$.

Since $\alpha_{j}$ is the binary representation of integer $j, m$ can be regarded as a permutation on $\left\{0,1, \ldots, 2^{k}-1\right\}$ and hence $\omega\left(\alpha_{j}\right)=\alpha_{i}$ can be equivalently written as $\omega(j)=i$. Let $\xi$ be the sequence of $f$. Then the first half of $\xi$ is specified by $1 \oplus\langle u, x\rangle$, while the second half by $\langle m(u), x\rangle$. To be more precise, the first half is (the concatenation of) $-\ell_{0},-\ell_{1}, \ldots,-\ell_{2^{k}-1}$, where each $\ell_{i}$ is the $i$ th row in $H_{k}$ and $-\ell_{i}$ means multiplying each component of $\ell_{i}$ by -1 . And the second half is $\ell_{m(0)}, \ell_{m(1)}, \ldots, \ell_{m\left(2^{k}-1\right)}$, a reordered version of $\ell_{0}, \ell_{1}, \ldots, \ell_{2^{k}-1}$ according to the permutation $m$ on $V_{k}$. Thus the sequence of $f$ takes the form of

$$
\xi=\left(-\ell_{0},-\ell_{1}, \ldots,-\ell_{2^{k}-1}, \ell_{m(0)}, \ell_{m(1)}, \ldots, \ell_{m\left(2^{k}-1\right)}\right) .
$$


Obviously $\xi$ contains an equal number of ones and minus ones. Hence $f$ is a balanced function on $V_{2 k+1}$. Using very similar arguments to those for the function $f$ on $V_{2 k}$ defined by (5) with attention to the fact that both $m(u)$ and $u \oplus m(u)$ are permutations, we can find out other properties of the function $f$ on $V_{2 k+1}$ defined by $(13)$. In particular, we have

Theorem 17. Let $f$ be the function on $V_{2 k+1}$ defined in (13). Then

(i) $f$ is balanced,

(ii) the nonlinearity of $f$ satisfies $N_{f} \geq 2^{2 k}-2^{k}$,

(iii) $f$ satisfies the propagation criterion with respect to $2^{2 k}-1$ nonzero vectors,

(iv) the sum-of-squares avalanche characteristic of $f$ satisfies $\sigma_{f}=2^{4 k+3}$,

(v) the absolute avalanche characteristic of $f$ satisfies $\Delta_{f} \leq 2^{k+1}$.

An important property of $f$ is that $\Delta_{f}$ matches the lower bound we conjectured at the end of Section 4. Comparing $\sigma_{f}=2^{4 k+3}=2 \cdot 2^{4 k+2}$ with $2^{4 k+2}$ and $2^{6 k+3}$, the upper and upper bounds respectively (see also Theorem 6 ), we can see that the sum-of-squares avalanche characteristic of the function is also extremely good. Again we conjecture that it achieves the lowest possible value for balanced functions on $V_{2 k+1}$.

It should be noted that since the total number of nonzero vectors with respect to which $f$ satisfies the propagation criterion is $2^{2 k}-1$, there are at most $2 k$ linearly independent ones among the vectors. Therefore, unlike the case on $V_{2 k}$, the function $f$ on $V_{2 k+1}$ constructed by (13) can not be transformed into an SAC-fulfilling one.

\section{Acknowledgments}

The authors would like to thank the anonymous referees for their comments that have helped in improving the presentation of this paper. This work was supported in part by the Australian Research Council (ARC) under the reference number A49232172 and by the Australian Telecommunications and Electronics Research Board (ATERB) under the reference numbers C010/058 and N069/412.

\section{References}

[AT90] C. M. Adams and S. E. Tavares. Generating and counting binary bent sequences. IEEE Transactions on Information Theory, IT-36 No. 5:1170-1173, 1990.

[Bea84] K. G. Beauchamp. Applications of Walsh and Related Functions with an Introduction to Sequency Functions. Microelectronics and Signal Processing. Academic Press, London, New York, Tokyo, 1984.

[BKPS93] L. Brown, M. Kwan, J. Pieprzyk, and J. Seberry. Improving resistance to differential cryptanalysis and the redesign of LOKI. In Advances in Cryptology - ASIACRYPT'91, volume 739, Lecture Notes in Computer Science, pages 36-50. Springer-Verlag, Berlin, Heidelberg, New York, 1993.

[BS93] E. Biham and A. Shamir. Differential Cryptanalysis of the Data Encryption Standard. Springer-Verlag, New York, Heidelberg, Tokyo, 1993.

[Dil72] J. F. Dillon. A survey of bent functions. The NSA Technical Journal, pages 191-215, 1972. (unclassified). 
[DT93] J. Detombe and S. Tavares. Constructing large cryptographically strong Sboxes. In Advances in Cryptology - AUSCRYPT'92, volume 718, Lecture Notes in Computer Science, pages 165-181. Springer-Verlag, Berlin, Heidelberg, New York, 1993.

[For89] R. Forré. The strict avalanche criterion: Special properties of boolean functions and extended definition. In Advances in Cryptology - CRYPTO'88, volume 403, Lecture Notes in Computer Science, pages 450-468. SpringerVerlag, Berlin, Heidelberg, New York, 1989.

[Gol82] S. W. Golomb. Shift Register Sequences. Laguna Hills, CA: Aegean Park, 1982 .

[Llo90] S. Lloyd. Counting functions satisfying a higher order strict avalanche criterion. In Advances in Cryptology - EUROCRYPT'89, volume 434, Lecture Notes in Computer Science, pages 64-74. Springer-Verlag, Berlin, Heidelberg, New York, 1990.

[Llo92] S. Lloyd. Counting binary functions with certain cryptographic properties. Journal of Cryptology, 5(2):107-132, 1992.

[Mit90] C. Mitchell. Enumerating boolean functions of cryptographic significance. Journal of Cryptology, 2(3):155-170, 1990.

[MS77] F. J. MacWilliams and N. J. A. Sloane. The Theory of Error-Correcting Codes. North-Holland, Amsterdam, New York, Oxford, 1977.

[Nyb91] K. Nyberg. Perfect nonlinear S-boxes. In Advances in Cryptology - EUROCRYPT'91, volume 547, Lecture Notes in Computer Science, pages 378-386. Springer-Verlag, Berlin, Heidelberg, New York, 1991.

[Nyb93] K. Nyberg. On the construction of highly nonlinear permutations. In $\mathrm{Ad}$ vances in Cryptology - EUROCRYPT'92, volume 658, Lecture Notes in Computer Science, pages 92-98. Springer-Verlag, Berlin, Heidelberg, New York, 1993.

[PGV91] B. Preneel, R. Govaerts, and J. Vandewalle. Boolean functions satisfying higher order propagation criteria. In Advances in Cryptology - EUROCRYPT'91, volume 547, Lecture Notes in Computer Science, pages 141-152. Springer-Verlag, Berlin, Heidelberg, New York, 1991.

[PLL $\left.{ }^{+} 91\right]$ B. Preneel, W. V. Leekwijck, L. V. Linden, R. Govaerts, and J. Vandewalle. Propagation characteristics of boolean functions. In Advances in Cryptology - EUROCRYPT'90, volume 437, Lecture Notes in Computer Science, pages 155-165. Springer-Verlag, Berlin, Heidelberg, New York, 1991.

[Rot76] O. S. Rothaus. On "bent" functions. Journal of Combinatorial Theory, Ser. A, 20:300-305, 1976.

[SMZ93] J. Seberry, X. M., and Y. Zhang. Highly nonlinear 0-1 balanced functions satisfying strict avalanche criterion. In Advances in Cryptology - AUSCRYPT'92, volume 718, Lecture Notes in Computer Science, pages 145-155. Springer-Verlag, Berlin, Heidelberg, New York, 1993.

[SZZ94a] J. Seberry, X. M. Zhang, and Y. Zheng. Improving the strict avalanche characteristics of cryptographic functions. Information Processing Letters, 50:3741, 1994 .

[SZZ94b] J. Seberry, X. M. Zhang, and Y. Zheng. Nonlinearly balanced boolean functions and their propagation characteristics. In Advances in Cryptology CRYPTO'93, volume 773, Lecture Notes in Computer Science, pages 49-60. Springer-Verlag, Berlin, Heidelberg, New York, 1994.

[SZZ94c] J. Seberry, X. M. Zhang, and Y. Zheng. Relationships among nonlinearity criteria. Presented at EUROCRYPT'94, 1994.

[SZZ94d] J. Seberry, X. M. Zhang, and Y. Zheng. Structures of cryptographic functions with strong avalanche characteristics. Asiacrypt'94, December 1994.

[SZZ95] J. Seberry, X. M. Zhang, and Y. Zheng. Nonlinearity and propagation characteristics of balanced boolean functions. To appear in Information and Computation, 1995. 
[Web85] A. F. Webster. Plaintext/ciphertext bit dependencies in cryptographic system. Master's Thesis, Department of Electrical Engineering, Queen's University, Ontario, Cannada, 1985.

[WT86] A. F. Webster and S. E. Tavares. On the design of S-boxes. In Advances in Cryptology - CRYPTO'85, volume 219, Lecture Notes in Computer Science, pages 523-534. Springer-Verlag, Berlin, Heidelberg, New York, 1986.

[YH89] R. Yarlagadda and J. E. Hershey. Analysis and synthesis of bent sequences. IEE Proceedings (Part E), 136:112-123, 1989. 